\title{
Two Level Centre of Gravity Computation -An Important Parameter for Offline Signature Recognition
}

\author{
Dipti Verma \\ Research scholar RCET, \\ Bhilai (C.G) India,
}

\author{
Sipi Dubey, PhD. \\ Dean (R\&D) RCET, \\ Bhilai, (C.G) India,
}

\begin{abstract}
This paper proposed feature extraction method based on centre of gravity for offline signature. Similar to other biometric measures, signatures have inherent variability and so pose a difficult recognition problem. Signature probably is one of the oldest biometric recognition methods, with high legal acceptance Signature has been a distinguishing feature for person identification through ages. Even today an increasing number of transactions, especially financial, are being authorized via signatures. In this paper, signature is preprocessed through binarization, cutting edges and thinning which provides more accurate platform for feature extraction methods. We have computed centre of gravity in two level by considering centre of gravity of all the characters separately instead of taking one common centre of gravity for entire signature and finally we would be able to built a system for signature recognition by taking mean values of all the centre of gravity values of various characters present in the signature.
\end{abstract}

\section{General Terms}

Image processing, Pattern Recognition, Authenticity.

\section{Keyword}

Static,dynamic, signature recognition, pre-processing, edge detection, centre of gravity.

\section{INTRODUCTION}

Biometrics is the science and technology of measuring analyzing biological data. In information technology, Biometrics refers to the technology that measure and analyzes human body characteristics for authentication purpose [1]. Human recognize each other by their various characteristics for ages. Biometrics offer automated method of identity verification or identification on the principle of measurable physiological or behavioral characteristic such as fingerprint or voice etc. Automatic signature verification is an active research field with many applications. There are two major categories: static and dynamic signature verification.

\section{DYNAMIC SIGNATURE}

In this mode, users write their signature in a digitizing tablet, which acquire the signature in real-time [6]. Dynamic recognition is also known as on-line recognition. On-line recognition means that the machine recognizes the handwriting as the user writes. It requires a transducer that captures the signature as it is written. The on-line system produces time information like acceleration (Speed of writing), retouching, pressure and pen movement [3].

\section{STATIC SIGNATURE}

In this mode, users write their signature on paper, digitize it through an optical scanner or a camera, and signature can be stored as an image form and the biometric system recognizes the signature analyzing its shape, this group is also known as off-line. Off-line handwriting recognition, is performed after the writing is complete. The data are captured at a later time by using an optical scanner to convert the image into a bit pattern. Off-line signature processing remains important since it is required in office automation systems. It is used for the validation of cheques, credit cards, contracts, historical documents, etc. Off-line have total 37 features like Centre of Gravity, Edges, curves etc. for authentication [1].

Offline signature recognition is an important form of biometric identification that can be used for various purposes [2]. Signatures are a socially accepted identification method and are commonly used in bank, credit-card transactions, and various business functions. Recognizing signatures can be used to verify identity and authenticate documents. Moreover, given a large collection of business documents relating to legal or intelligence investigations, signatures can be used to identify documents authored or authorized by specific individuals. This is an important form of indexing that can be used in the exploration of the data [3].

Offline signature recognition is a challenging task due to normal variability in signatures and the fact that dynamic information regarding the pen path is not available [4] Moreover, training data are normally limited to only a small number of signatures per subject. The application of offline signature recognition has been studied in the context of biometrics to perform authentication, and, more recently, in the context of indexing and retrieval of document images in a large database. This, however, comes at the cost of simplifying the actual signature data. Related to offline signature recognition is the problem of shape matching. Shape matching is normally treated by determining and matching key points so as to avoid the problems associated with the detection and parameterization of curves. It should be noted, however, that the use of feature points [5].

The application areas for signature recognition include all applications where handwritten signatures are already used such as in bank transactions, credit card operations or authentication of a document with important instructions or information. The purpose of the signature recognition process is to identify the writer of a given sample, while the purpose of the signature verification process is to confirm or reject the sample.

Some of the error occurs in the biometric signature recognition are:

\subsection{False Rejection (FR)}

The legitimate user is rejected because the system does not find the user's current biometric data similar enough to the master template stored in the database [3].Calculated in terms of FRR (false rejection rate) the ratio of the number of false 
rejections divided by the number of total identification attempts.

\subsection{False Acceptance (FA)}

An imposer is accepted as a legitimate user because the system finds the imposter's biometric data similar enough to master template of a legitimate user [3].calculated in terms of FAR (false acceptance rate) the ratio of the number of false acceptances divided by the number of total identification attempts.

\subsection{Types of Forgeries}

Apart from the FA and FR according to the authentication procedure, many other different errors can occur in signature recognition process like forgeries; there are three types of forgery, random, simple and simulated [11]. In the first type, the forger has no previous knowledge of the signature and the style of the forged signature differs from the original. Therefore, random forgeries are easily identified. In the second type signatures are based on an assumption of how the signature looks like by knowing the name of the signer and in the third type, the forger knows the signature well and has the ability to simulate or copy it. Therefore, the simulated signature is very similar to the original one, making it much more difficult to verify the forgery [13].

\section{SIGNATURE DATABASE}

The signature samples were acquired from the 50 individuals all these signatures were scanned and stored in the database record so that we can use these signatures for the preprocessing steps and feature extraction method. In this paper 15 signature images samples $S \_1$ to $S \_15$ are shown below table1.

Table 1.Sample database

Raulut

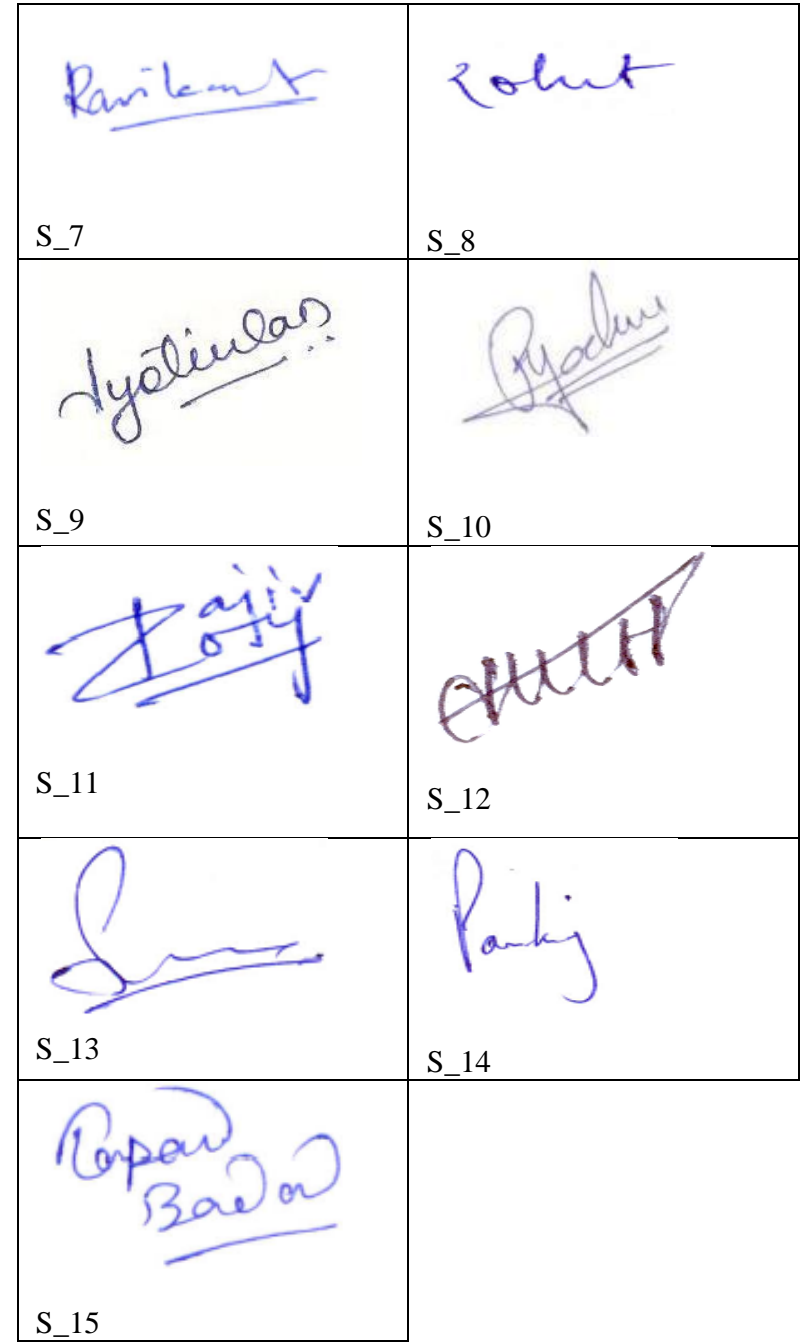

\section{ALGORITHM DESIGNED FOR THE USED METHODOLOGY}

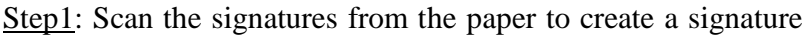
image database.

Step2: Resize the scanned image and is converted into gray scale image, and then it is thinned so that its important area can be highlighted.

Step3: Morphological operations are performed, it gradually enlarge the boundaries of regions of the foreground pixels.

Step4: Determine the edges of shapes in an image where edges are in white on black background. Area of the image is filtered which removes small dots and isolated pixels, as it effects the local features of the signature.

Step5: Then Centre of gravity is extracted from each signature in two levels.Level-1 gives the centre of gravity values of individual connected character present in the equation and Level- 2 calculates the final centre of gravity value obtained by calculating mean of the level-1 output values, as similar images have central points of gravity which are approximately the same for their similar segments or parts. 


\section{PREPROCESSING}

Preprocessing means standardization of images which is important before feature extraction, all signatures are binarized, thinned. And its size standardization is performed.Step1,2,3 of the designed algorithm are the preprocessing steps. A wide variety of devices capturing signature causes the need to normalize an input image of signature so called preprocessing [7]. Some times may possible that people do not always sign documents in exactly the same manner like the angle at which they sign may be different due to seating position or due to hand placement on the writing surface. For this reason the original signature should be appropriate formatted and preprocessed. In this paper three steps are practiced for preprocessing of static signature that is binarization, cutting edges and thinning.

\subsection{Binarization}

In binarization method we reduce the amount of image information (removing color and background), so the output image is in black-white. The black-white type of the image is much more easily to further processing [7].

$$
\mathrm{Q}=\frac{S}{X * Y}
$$

Where: $S$ is the sum values of all image's pixels and $X, Y$ is horizontal and vertical size of the signature image, respectively. Value of the each image pixel is compared to value of $Q$ : if this value is greater than $Q$, then appropriate pixel is set to the white colour, otherwise this pixel is set to the black colour.

\subsection{Cutting edges}

By cutting edges size of the image is reduced. In this procedure unnecessary signature areas are removed or we can say that we find the maximum/minimum value of the $\mathrm{X}$ and $\mathrm{Y}$ coordinates of the signature and then the image is cut to the signature size. It allows reducing the total number of the pixels in the analyzed image [7].

\subsection{Thinning}

Thinning allows us to form a region based shape of the signature here thresholding process is used. It should be noticed that main features of the object are protected [7]. This eliminates the effect of different line thicknesses resulting from the use of different writing pens, as the result of thinning skeletonized signature image of 1-pixel shape is obtained. Pavlidis algorithm is used for obtaining thinned image [8]
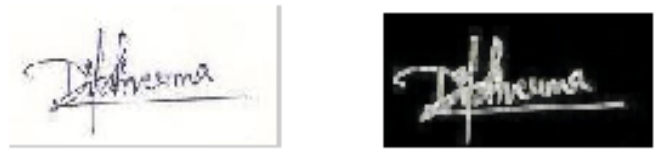

Figure 1: (a) Binarized image

(b). Preprocessed image

\section{FEATURE EXTRACTION}

The feature selection and extraction play a major role in all pattern recognition systems. It is preferable to extract those features which will enable the system to correctly discriminate one class from the other [6]. As true samples and forgeries are very similar in most cases, it is very important to extract an appropriate feature set to be used in discriminating between genuine and forged signatures. It is a fact that any forgery must contain some deviations if compared to the original model and that a genuine sample is rarely identical to the model although there must be similarities between them [8] Such similarities make it possible to determine the features needed for the verification process.

Features are effective in the verification process as they show a relatively high rate of correctness since they give more importance to pixel positions and are less sensitive to noise [8]. Feature extraction is a gathering of characteristic data which provides an output result as a set of the unique information about the signature.

Process for feature extraction: firstly we took height and length only as the features to be extracted for matching signature in a data base. 20 signatures of different persons were taken for testing but it gave error because it is possible that length and height of the signature may vary due to change in speed, angle of doing signature and therefore selected centre of gravity as an important feature so that the verification procedure get easily done[9].

\subsection{Edge detection- Laplacian of Gaussian}

The Laplacian is a 2-D isotropic measure of the 2nd spatial derivative of an image. The Laplacian of an image highlights regions of rapid intensity change and is therefore often used for edge detection. The Laplacian is often applied to an image that has first been smoothed with something approximating a Gaussian smoothing filter in order to reduce its sensitivity to noise, and hence the two variants will be described together here[11]. The operator normally takes a single gray level image as input and produces another gray level image as output. The Laplacian $L(x, y)$ of an image with pixel intensity values $I(x, y)$ is given by:

$\mathrm{L}(\mathrm{x}, \mathrm{y})=$

$\frac{\partial^{2} \mathrm{I}}{\partial \mathrm{x}^{2}}+\frac{\partial^{2} \mathrm{I}}{\partial \mathrm{y}^{2}}$

This can be calculated using a convolution filter. Since the input image is represented as a set of discrete pixels, we have to find a discrete convolution kernel that can approximate the second derivatives in the definition of the Laplacian.

\subsection{Centre of Gravity}

In this step centre of gravity is calculated-it is a point $G(x g$, $y g$ ) where appropriate lines A and B are crossing. These lines divide the signature image into vertical and horizontal regions where number of pixels in those regions is the same [10]. The coordinates $(x g, y g)$ are obtained based on analysis of the vertical and horizontal projection arrays Nvert and Nhori, respectively. The value of the coordinate $x g$ is equal to such index $k x$ of the cell of the Nvert array, for which the next condition is fulfilled [12], here centre of gravity is calculated in two levels, in the first level we calculate the centre of gravity of each character present in the signature, figure. 3 shows the level-1 image which contains red marks on each connected characters specifies the centre of gravity values and in the second level mean is calculated from the output of level-1.

$$
\begin{gathered}
\sum_{i=0}^{K_{x}-1} N_{\text {vert }}[i]<\frac{\sum_{i=0}^{255} N_{\text {vert }}[i]}{2} \wedge \sum_{i=0}^{k_{v}} N_{\text {vert }}[i] \\
\geq \frac{\sum_{i=0}^{255} N_{\text {vert }}[i]}{2}
\end{gathered}
$$




$$
\begin{gathered}
\sum_{i=0}^{K_{Y}-1} N_{\text {hori }}[i]<\frac{\sum_{i=0}^{255} N_{\text {hori }}[i]}{2} \wedge \sum_{i=0}^{k_{v}} N_{\text {hori }}[i] \\
\geq \frac{\sum_{i=0}^{255} N_{\text {hori }}[i]}{2}
\end{gathered}
$$

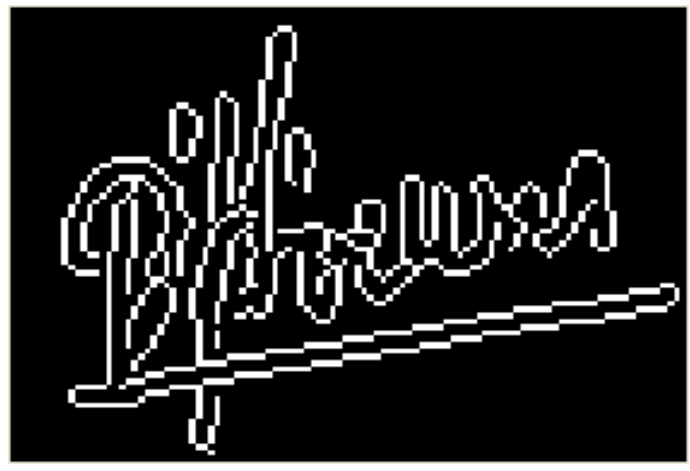

Figure 2: Image obtained by edge detection

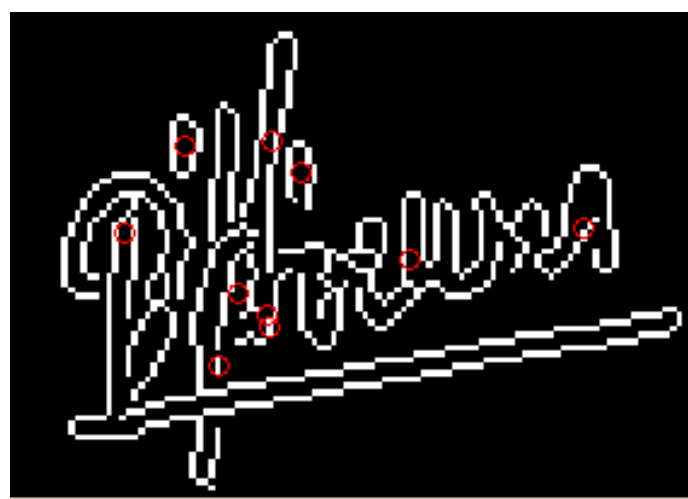

Figure 3: Centre of gravity level-1 image

\section{RESULT}

50 individual signatures are taken for verification process and preprocessing steps are performed on these signatures so that further processing gets easier and efficient. For the verification process different features are extracted using various methods, and on the basis of these features matching of the signatures is performed, this paper focuses on center of gravity as an important feature which provides the more accurate values for the matching process, the values obtained after two level centre of gravity computation is shown through graphical representation in figure. 4 and as an outcome of this paper the differences between the centre of gravity values obtained for first 15 signature sample images is shown in the table 3.
In order to know the variations among the various signatures; centre of gravity value is taken into the consideration and the differences of each sample signature with all other samples is calculated, on observing the table 3 , it is clear that every signature is having certain variation from other with reference to the value obtained. The proposed method gives $80-90 \%$ influenced accuracy by the features considered in verification process. Comparative study between existing techniques and proposed method is shown through table 2, which shows the various methods with the combination of features taken and influences of features observed on the verification techniques.

Table 2.Comparison between existing techniques and proposed method.

\begin{tabular}{|l|l|l|}
\hline Methods & $\begin{array}{l}\text { Combination of } \\
\text { Features }\end{array}$ & $\begin{array}{l}\text { Features } \\
\text { influence on } \\
\text { verification } \\
\text { process }\end{array}$ \\
\hline $\begin{array}{l}\text { SVM(support } \\
\text { vector machine) } \\
\text { based verification }\end{array}$ & $\begin{array}{l}\text { Grid, mask, and global } \\
\text { features }\end{array}$ & $80-85 \%$ \\
\hline $\begin{array}{l}\text { Compact three } \\
\text { stage method }\end{array}$ & $\begin{array}{l}\text { Proportion factor, } \\
\text { horizontal and vertical } \\
\text { projection, single level } \\
\text { centre of gravity, }\end{array}$ & $55-65 \%$ \\
\hline $\begin{array}{l}\text { Offline signature } \\
\text { verification using } \\
\text { NN (neural } \\
\text { network) }\end{array}$ & $\begin{array}{l}\text { Eccentricity, skewness, } \\
\text { kurtosis, oriantation }\end{array}$ & $65-80 \%$ \\
\hline Proposed method & $\begin{array}{l}\text { Edges, two level centre of } \\
\text { gravity }\end{array}$ & $80-90 \%$ \\
\hline
\end{tabular}

\section{CONCLUSION AND FUTURE WORK}

Centre of gravity is an important parameter or feature used for the matching process of signatures. The existing techniques computes a single level value for centre of gravity, where as in this paper the centre of gravity value is more refined and calculated in two levels providing high accurate value to distinguish signatures easily. As matching process is totally dependent on accurate value of features considered: further some more refined feature values can be combined with the two level centre of gravity computation so that verification process gets more accurate and authenticity can be increased. 


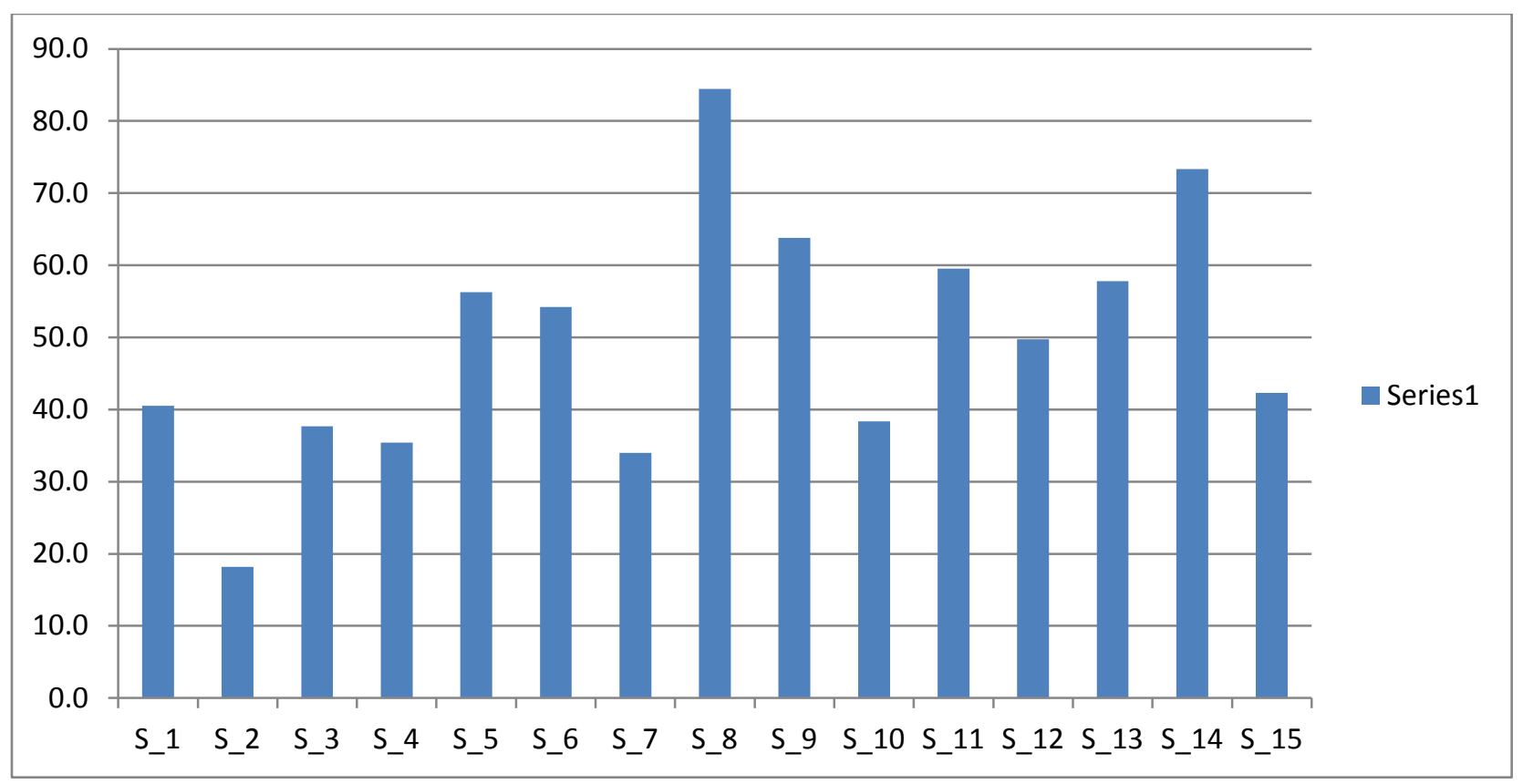

Figure 4: Graphical representaion of the centre of gravity values obtained from 2level centre of gravity computation.

Table 3.Differences between the values of center of gravity for each signature.

\begin{tabular}{|c|c|c|c|c|c|c|c|c|c|c|c|c|c|c|c|c|}
\hline & & S_1 & S_2 & S_3 & S_4 & S_5 & S_6 & S_7 & S_8 & S_9 & S_10 & S_11 & S_12 & S_13 & S_14 & S_15 \\
\hline & & 40.5 & 18.2 & 37.7 & 35.4 & 56.3 & 54.2 & 34.0 & 84.4 & 63.8 & 38.4 & 59.5 & 49.7 & $\mathbf{5 7 . 8}$ & 73.4 & 42.3 \\
\hline S_1 & 40.5 & 0.0 & 22.3 & 2.8 & 5.1 & $\begin{array}{r}- \\
15.8\end{array}$ & $\begin{array}{r}- \\
13.7\end{array}$ & 6.5 & $\begin{array}{r}- \\
43.9 \\
\end{array}$ & $23 . \overline{-}$ & 2.1 & 19.0 & -9.2 & $\begin{array}{r}- \\
17.3\end{array}$ & $\begin{array}{r}- \\
32.9 \\
\end{array}$ & -1.8 \\
\hline S_2 & 18.2 & $\begin{array}{r}- \\
22.3 \\
\end{array}$ & 0.0 & $\begin{array}{r}- \\
19.5 \\
\end{array}$ & $17 . \overline{-}$ & $\begin{array}{r}- \\
\end{array}$ & $\begin{array}{r}- \\
36.0 \\
\end{array}$ & $\begin{array}{r}- \\
15.8 \\
\end{array}$ & $\begin{array}{r}- \\
66.2 \\
\end{array}$ & $\begin{array}{r}- \\
45.6 \\
\end{array}$ & -20.2 & $\begin{array}{r}- \\
41.3 \\
\end{array}$ & 31.5 & $\begin{array}{r}- \\
39.6 \\
\end{array}$ & 55.2 & $\begin{array}{r}- \\
24.1 \\
\end{array}$ \\
\hline S_3 & 37.7 & -2.8 & 19.5 & 0.0 & 2.3 & $\begin{array}{r}- \\
18.6\end{array}$ & $\begin{array}{r}-\overline{-} \\
16.6 \\
\end{array}$ & 3.7 & $\begin{array}{r}- \\
46.8 \\
\end{array}$ & $\begin{array}{r}- \\
26.1\end{array}$ & -0.7 & 21.8 & $\begin{array}{r}- \\
12.1\end{array}$ & $\begin{array}{r}- \\
20.1\end{array}$ & $\begin{array}{r}- \\
35.7 \\
\end{array}$ & -4.6 \\
\hline S_4 & 35.4 & -5.1 & 17.2 & -2.3 & 0.0 & 20.9 & 18.8 & 1.4 & $49.0^{-}$ & 28.4 & -3.0 & $24 . \overline{1}$ & 14.3 & 22.4 & 38.0 & -6.9 \\
\hline S_5 & 56.3 & 15.8 & 38.1 & 18.6 & 20.9 & 0.0 & 2.1 & 22.3 & 28.2 & -7.5 & 17.9 & -3.2 & 6.5 & -1.5 & 17.1 & 14.0 \\
\hline S_6 & 54.2 & 13.7 & 36.0 & 16.6 & 18.8 & -2.1 & 0.0 & 20.2 & 30.2 & -9.6 & 15.8 & -5.3 & 4.5 & -3.6 & 19.1 & 11.9 \\
\hline S_7 & 34.0 & -6.5 & 15.8 & -3.7 & -1.4 & $22.3^{-}$ & $20.2^{-}$ & 0.0 & 50.4 & 29.8 & -4.4 & 25.5 & 15.7 & $23 . \overline{8}$ & $\begin{array}{r}- \\
39.4 \\
\end{array}$ & -8.3 \\
\hline S_8 & 84.4 & 43.9 & 66.2 & 46.8 & 49.0 & 28.2 & 30.2 & 50.4 & 0.0 & 20.6 & 46.1 & 24.9 & 34.7 & 26.6 & 11.1 & 42.1 \\
\hline S_9 & 63.8 & 23.3 & 45.6 & 26.1 & 28.4 & 7.5 & 9.6 & 29.8 & 20.6 & 0.0 & 25.4 & 4.3 & 14.1 & 6.0 & -9.6 & 21.5 \\
\hline S_10 & 38.4 & -2.1 & 20.2 & 0.7 & 3.0 & 17.9 & $\begin{array}{r}- \\
15.8 \\
\end{array}$ & 4.4 & $\begin{array}{r}- \\
46.1\end{array}$ & $\begin{array}{r}- \\
25.4\end{array}$ & 0.0 & $\begin{array}{r}- \\
21.1\end{array}$ & 11.4 & $\begin{array}{r}- \\
19.4\end{array}$ & $\begin{array}{r}- \\
35.0\end{array}$ & -3.9 \\
\hline S_11 & 59.5 & 19.0 & 41.3 & 21.8 & 24.1 & 3.2 & 5.3 & 25.5 & $24.9^{-}$ & -4.3 & 21.1 & 0.0 & 9.8 & 1.7 & $13.8^{-}$ & 17.2 \\
\hline S_12 & 49.7 & 9.2 & 31.5 & 12.1 & 14.3 & -6.5 & -4.5 & 15.7 & $\begin{array}{r}- \\
34.7 \\
\end{array}$ & $\begin{array}{r}- \\
14.1\end{array}$ & 11.4 & -9.8 & 0.0 & -8.1 & 23.6 & 7.4 \\
\hline S_13 & $\mathbf{5 7 . 8}$ & 17.3 & 39.6 & 35.7 & 22.4 & 1.5 & 3.6 & 23.8 & $\begin{array}{r}- \\
26.6 \\
\end{array}$ & -6.0 & 19.4 & -1.7 & 8.1 & 0.0 & $15.6^{-}$ & 15.5 \\
\hline S_14 & 73.4 & 32.9 & 55.2 & 35.7 & 38.0 & 17.1 & 19.1 & 39.4 & $11 . \overline{1}$ & 9.6 & 35.0 & 13.8 & 23.6 & 15.6 & 0.0 & 31.0 \\
\hline S_15 & 42.3 & 1.8 & 24.1 & 4.6 & 6.9 & 14.0 & 11.9 & 8.3 & 42.1 & 21.5 & 3.9 & 17.2 & -7.4 & 15.5 & 31.0 & 0.0 \\
\hline
\end{tabular}




\section{REFERENCES}

[1] Z.Riha and V.Matyas, "Biometric Authentication System”, FIMU RS-200-08, pp. 4-44, 2000

[2] G.Agam and S.Suresh,"Warping based Offline Signature recognition, "Information Forensics and Security, Vol.2, no.3, pp.430-437, 2007.

[3] F.Z Marcos, "signature recognition state-of-the-art", IEEE A\&E SYSTEMS MAGZINE JULY 2005, page: 28-32.

[4] J.wen, B.fang, and T.jhang, "offline signature verification: a new rotation invariant approach", pp 3583-3586.

[5] B.Jayasekara, and A.Jayasiri,L.Udawatta, "An Evolving Signature Recognition System" first International Conference on Industrial and Information Systems, ICIIS 2006, 8 - 11 August 2006, Sri Lanka,pp529-534

[6] J.Zheng, and G.Zhu, "On-Lin Handwriting Signature Recognition Based on Wavelet Energy Feature Matching", proceedings of the 6th World Congress on Intelligent Control and Automation, June 21 - 23, 2006, Dalian, China pp9885-9888.

[7] Dipti verma, pradeep mishra, tapas badal, "A Preprocessing And Feature Extraction Method For Static Signature Recognition Using Hough Transform And Wavelet Analysis" ICCET'10-international conference on computer engineering and technology
International Journal of Computer Applications (0975 - 8887) Volume 54-No.10, September 2012

[8] T.Pavlidis. "A thinning algorithm for discrete binary images. Computer Graphics and Image Processing", 1980, 13: 142-157.

[9] F.Nouboud and R.Plamondon, "Global Parameters and Curves for Off-Line Signature Verification",Workshop On Frontiers in Handwriting recognition Taiwan, 1994; pp. 145-155

[10] P.Porwik, "The compact three stage method of the signature recognition", proceeding on $6^{\text {th }}$ International conference on computer Information system and Industrial Management applications, 2007.

[11] EdsonJ.R.Justino,F.Bortolozzi,R.Sabourn "Offline Signature Verification Using Hmm For Random, Simple and Skilled Forgeries",Document analysis and recognition. Proceedings of $6^{\text {th }}$ international conference 2001

[12] Maya V. Karki, K. Indira, Dr. S. Sethu Selvi "Offline signature recognition and verification using Neural Network" proceeding of the International Conference on computational Intelligence and Multimedia Application,2007

[13] Suhail M. Odeh and Manal Khalil "Off-line signature verification and recognition: Neural Network Approach "in International Conference On Innovations In Intelligent Systems And Applications (INISTA ) 2011. 